\title{
Long wavelength identification of microcalcifications in breast cancer tissue using a quantum cascade laser and upconversion detection
}

Tseng, Yu-Pei; Bouzy, P.; Stone, N.; Pedersen, Christian; Tidemand-Lichtenberg, Peter

Published in:

Proceedings of SPIE

Link to article, DOI:

$10.1117 / 12.2290928$

Publication date:

2018

Document Version

Publisher's PDF, also known as Version of record

Link back to DTU Orbit

Citation (APA):

Tseng, Y-P., Bouzy, P., Stone, N., Pedersen, C., \& Tidemand-Lichtenberg, P. (2018). Long wavelength identification of microcalcifications in breast cancer tissue using a quantum cascade laser and upconversion detection. In Proceedings of SPIE [104900F] SPIE - International Society for Optical Engineering. Proceedings of SPIE - The International Society for Optical Engineering https://doi.org/10.1117/12.2290928

\section{General rights}

Copyright and moral rights for the publications made accessible in the public portal are retained by the authors and/or other copyright owners and it is a condition of accessing publications that users recognise and abide by the legal requirements associated with these rights.

- Users may download and print one copy of any publication from the public portal for the purpose of private study or research.

- You may not further distribute the material or use it for any profit-making activity or commercial gain

- You may freely distribute the URL identifying the publication in the public portal 


\section{Long wavelength identification of microcalcifications in breast cancer tissue using a quantum cascade laser and upconversion detection}

Y. P. Tseng, P. Bouzy, N. Stone, C. Pedersen, P. Tidemand-Lichtenberg

Y. P. Tseng, P. Bouzy, N. Stone, C. Pedersen, P. Tidemand-Lichtenberg, "Long wavelength identification of microcalcifications in breast cancer tissue using a quantum cascade laser and upconversion detection ," Proc. SPIE 10490, Biomedical Vibrational Spectroscopy 2018: Advances in Research and Industry, 104900F (13 February 2018); doi: 10.1117/12.2290928 


\title{
Long wavelength identification of microcalcifications in breast cancer tissue using a quantum cascade laser and upconversion detection
}

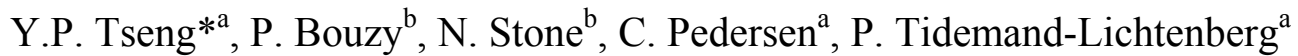 \\ a Technical university of Denmark, DTU Fotonik, Frederiksborgvej 399, 4000 Roskilde, Denmark; \\ ${ }^{\mathrm{b}}$ School of Physics and Astronomy, University of Exeter, EX4 4QL, England
}

\begin{abstract}
Spectral imaging in the long-wave infrared regime has great potential for medical diagnostics. Breast cancer is the most common cancer amongst females in the US. The pathological features and the occurrence of the microcalcifications are still poorly understood. However, two types of microcalcifications have been identified as unique biomarkers: type I consisting of calcium oxalate (benign lesions) and type II composed of hydroxyapatite (benign or invasive lesions). In this study, we propose a new approach based on vibrational spectroscopy that is non-destructive, label-free and chemically specific for breast cancer detection. Long-wave infrared spectroscopy combining quantum cascade lasers (QCL) and upconversion detection, offer to improve signal-to-noise ratios compared to standard long-wave infrared spectroscopy. We demonstrated long-wave identification of synthetic samples of carbonated hydroxyapatite and of microcalcification in breast cancer tissue using upconversion detection. Absorbance spectra and upconverted images of in situ breast cancer biopsy are compared with that of Fourier-transform infrared (FTIR) spectroscopy.
\end{abstract}

Keywords: Upconversion, Infrared image, Medical optics

\section{INTRODUCTION}

Breast cancer is the most commonly diagnosed cancer amongst females in the USA and comes in second place as the most frequent cause of death from cancer after lung cancer. In 2017, around 41,000 women are expected to die from breast cancer in the USA [1]. In order to increase the survival rate, an early diagnosis is important. In this context, the microcalcifications are the unique early marker for breast cancer detection. They are characterized by abnormal calcium deposits in mammary gland. Mammography is commonly used to screen populations for breast cancers. Unfortunately, this technique is not able to discriminate between microcalcifications from a benign or a malignant lesion [2].

Two types of microcalcifications are observed in the breast: type I consisting of calcium oxalate (benign lesions) and type II composed of calcium hydroxyapatite (proliferative lesions and invasive cancer). In biological tissues, hydroxyapatite (HAP) is not a pure compound but contains within its lattice around 5-6\% of carbonate $\left(\mathrm{CO}_{3}{ }^{2-}\right)$ substitution [3]. Two types of substitution have been observed for the intercalation of carbonate in the HAP lattice: type A when $\mathrm{CO}_{3}{ }^{2-}$ replaces the $\mathrm{OH}^{-}$ions, and type $\mathrm{B}$ when $\mathrm{CO}_{3}{ }^{2-}$ replaces the phosphate $\left(\mathrm{PO}_{4}{ }^{3-}\right)$ ions [4]. In order to understand the pathological mineralization process in biological samples, in particular in breast cancer microcalcification, Fourier Transform Infrared (FTIR) spectroscopy can be used to measure the spectral features of breast cancer tissue and HAP synthetic carbonated samples [3]. FTIR system is equipped with standard infrared detectors, such as Mercury cadmium telluride (MCT) detector and Focal Plane Arrays (FPA). However, direct infrared detectors generally suffer from inherent dark noise and require cooling for low-noise infrared detection [5].

In recent years frequency upconversion has been revitalized as an alternative approach to direct detection of infrared radiation [6,7]. Converting long-wave infrared emission into the visible/near infrared regime via nonlinear frequency conversion allows for the use of silicon based detectors having signal-to-noise ratio, orders of magnitude better than that of direct infrared detectors. Moreover, silicon detectors are low noise, fast, sensitive, and cost efficient compared to their mid-infrared counterpart; although this needs to be balanced with losses in signal during the frequency upconversion process.

*yupts@fotonik.dtu.dk

Biomedical Vibrational Spectroscopy 2018: Advances in Research and Industry, edited by

Anita Mahadevan-Jansen, Wolfgang Petrich, Proc. of SPIE Vol. 10490, 104900F

(C) 2018 SPIE · CCC code: 1605-7422/18/\$18 · doi: 10.1117/12.2290928 
In this paper, we demonstrate a new approach for non-destructive, label-free and chemically specific vibrational spectroscopy based on frequency upconversion. The combination of a high brightness infrared source i.e. a quantum cascade laser (QCL), an $x$ - and $y$-axis scanning microscope, and upconversion detection potentially offers an improved signal-to-noise ratio compared to standard infrared spectroscopy imaging systems in the long-wave infrared (LWIR) regime. Raster scanning at discrete wavelengths is used to form chemical images at selected wavelengths according to the spectral features in breast cancer tissue.

\section{EXPERIMENTAL SETUP}

The setup is illustrated in Figure 1. A QCL is used as a widely tunable, narrow linewidth, long-wave infrared source spanning from 9.43 to $12 \mu \mathrm{m}$, delivering $50 \mathrm{~ns}$ pulses with a temporal separation of $1 \mu \mathrm{s}$. The LWIR beam is coupled into a homemade scanning microscope allowing for mechanically stable scanning of the sample. The sample is placed between two ZnSe lenses of focal lengths $\mathrm{f}_{1}=\mathrm{f}_{2}=20 \mathrm{~mm}$ giving a LWIR beam waist (radius) of approx. $48 \mu \mathrm{m}$ in the sample plane. The beam diameter determines the spatial resolution in the raster scanning approach. The LWIR beam is then guided and refocused into the upconversion unit in which the beam $(100 \mu \mathrm{m}$ radius $)$ is mixed with a near infrared (NIR) laser beam (100 $\mu \mathrm{m}$ radius) inside a nonlinear crystal optimized for sum frequency generation. An $8 \mathrm{~W}$ diode laser is used to pump a Nd: $\mathrm{YVO}_{4}$ laser generating the $1064 \mathrm{~nm}$ single-pass mixing field, giving a maximum output power of $3 \mathrm{~W}$ in continuous-wave operation. The sum frequency generation (SFG) is realized in a $5 \times 5 \times 10 \mathrm{~mm}^{3} \mathrm{AgGaS}_{2}$ crystal. The $\mathrm{AgGaS}_{2}$ crystal is cut at $\theta=43.3^{\circ}(\varphi=0)$ for collinear type II phase-matching $\left(e_{\mathrm{LWIR}}+o_{\mathrm{NIR}} \rightarrow e_{\mathrm{Up}}\right)$. The $\mathrm{AgGaS}_{2}$ crystal is mounted on a piezo controlled rotation stage for angle tuned birefringent phase-matching, converting the LWIR signal to the 950 to $980 \mathrm{~nm}$ range for detection using a silicon power meter.

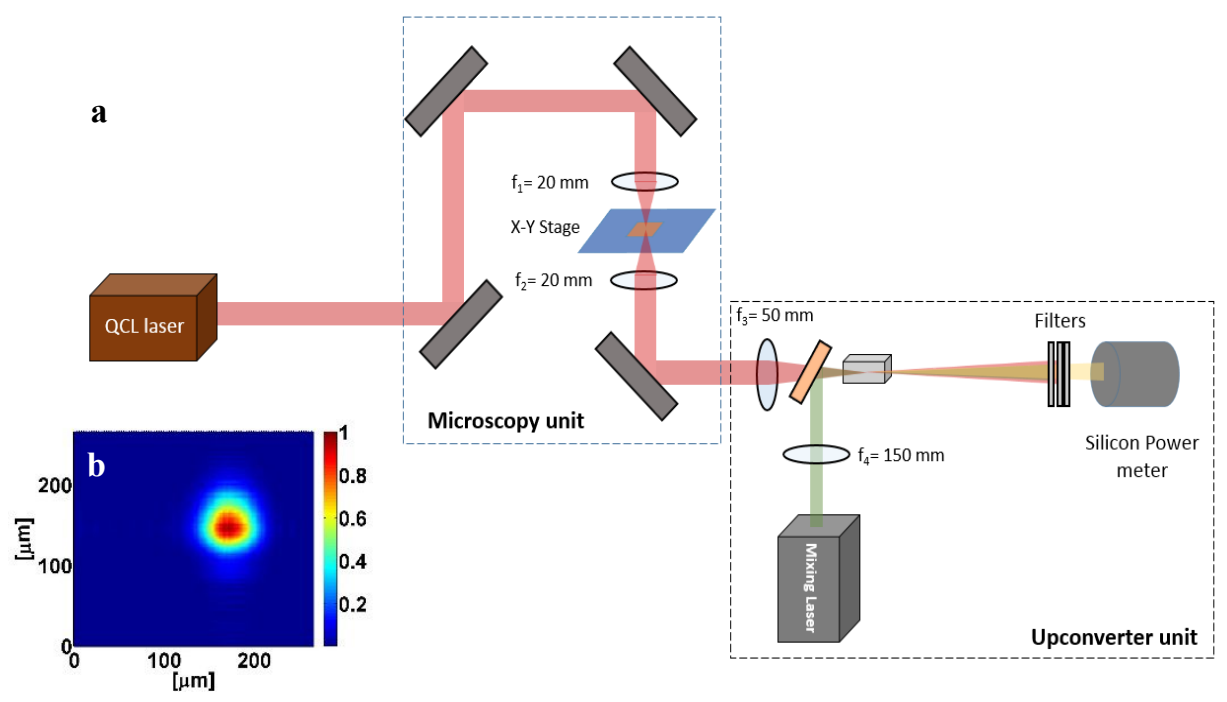

Figure 1: (a) Schematic layout of LWIR light source, scanning microscopy unit, and upconversion detection unit based on sum frequency generation (b) beam profile at the focus of LWIR light source.

\section{MATERIALS AND METHODS}

\subsection{KBr pellets with Hydroxyapatite samples}

In order to improve the understanding of the pathological mineralization process in breast cancer, we first examine synthetic samples of carbonated hydroxyapatite (HAP) using vibrational spectroscopy. For this, we have compared the performance of a commercial FTIR system and the developed upconversion system. KBr pellets of different standard minerals, provided by Prof Mary Tecklenburg, Central Michigan University, USA, containing carbonate substitution of $1.24,2.92,4.43,5.24,7.52$ and $8.12 \%$ are investigated according to the following protocol: $99 \mathrm{mg}$ of $\mathrm{KBr}$ powder, was 
added to $0.5 \mathrm{mg}$ of Hydroxyapatite (HAP) and then mixed and grounded together by a mortar and pestle. The mixture was transferred into a hydraulic press and compressed for 30 minutes with a pressure of 10 tons. A pellet containing only $100 \mathrm{mg}$ of $\mathrm{KBr}$ has been used to obtain a background spectrum.

\subsection{Breast cancer sample}

An in situ breast cancer biopsy with microcalcifications, provided by Dr Catherine Kendall, Gloucestershire Royal Hospitals NHS Foundation Trust, was analyzed using the upconversion system. We compared the images and spectra of this sample with using an FTIR Agilent system and upconversion. The sample came from a patient who had undergone a biopsy for mammographically suspicious breast lesions, and it was selected from the histopathological report. The biopsy was embedded in paraffin, two adjacent sections of $3 \mu \mathrm{m}$ thickness were analyzed: one section mounted on Barium fluoride $\left(\mathrm{BaF}_{2}\right)$ without staining was analyzed using the FTIR and upconversion systems, and one section stained with Hematoxylin Eosin (H\&E) for the histological analysis as a 'gold standard' for diagnosis.

\subsection{Micro-FTIR system}

The breast cancer biopsies were analyzed using Agilent 670 FTIR spectrometer coupled with an Agilent 620 FTIR microscope, equipped with a 15X Cassegrain objective and a Mercure Cadmium Telluride - Focal Array Plane (MCTFPA) detector comprising of 128x128 pixels and a Globar source. Spectra were recorded with a spectral resolution of 4 $\mathrm{cm}^{-1}$, a spectral range comprised between $3900-800 \mathrm{~cm}^{-1}$ and a spatial resolution of $5.5 \mu \mathrm{m}$. Each spectrum was obtained by averaging 64 scans and a background spectrum was also recorded with 256 scans. The FTIR spectra were collected using Agilent ResolutionPro software.

The $\mathrm{KBr}$ pellet containing HAP were analyzed in single-point mode using an Agilent 670 FTIR spectrometer coupled with an Agilent 620 FTIR microscope, equipped with a 15X cassegrain objective and a MCT detector. Spectra were recorded in the spectral range between $4000-700 \mathrm{~cm}^{-1}$, with a spectral resolution of $4 \mathrm{~cm}^{-1}$. Each spectrum was obtained by averaging 32 scans and a background spectrum with 64 scans.

\section{RESULTS AND DISCUSSION}

\section{1 $\mathrm{KBr}$ pellets with Hydroxyapatite samples}

Absorption spectra of carbonated hydroxyapatite samples were recorded using FTIR system from 4000 to $700 \mathrm{~cm}^{-1}$ with a spectral resolution of $4 \mathrm{~cm}^{-1}$, are shown in Figure 2(a). Phosphate absorption peaks are found in the range of 1020 to 1040 and at $962 \mathrm{~cm}^{-1}$, and a carbonate peak is observed at $875 \mathrm{~cm}^{-1}$ using the FTIR system. Figure 2(b) shows the spectrum of HAP samples with 7.52 and $2.92 \%$ carbonate substitution measured using the upconversion system shown in Figure 1. The spectra were recorded from 1066 to $830 \mathrm{~cm}^{-1}$ in steps of $4 \mathrm{~cm}^{-1}$, and averaged by 5 scans for each sample. The measured spectra are normalized similar to FTIR absorbance spectra in Figure 2(a). The HAP spectra shown in Figure 2(b) were measured at a single point, with beam size of $48 \mu \mathrm{m}$ radius, at the sample pellets. Absorption peaks in phosphate and carbonate bands are observed in smoothed spectrum (not shown) at 1044, 964 and $875 \mathrm{~cm}^{-1}$ in the HAP $7.52 \%$ carbonate substitution, and at 1044, 964 and $882 \mathrm{~cm}^{-1}$ in the HAP $2.92 \%$ carbonate substitution.
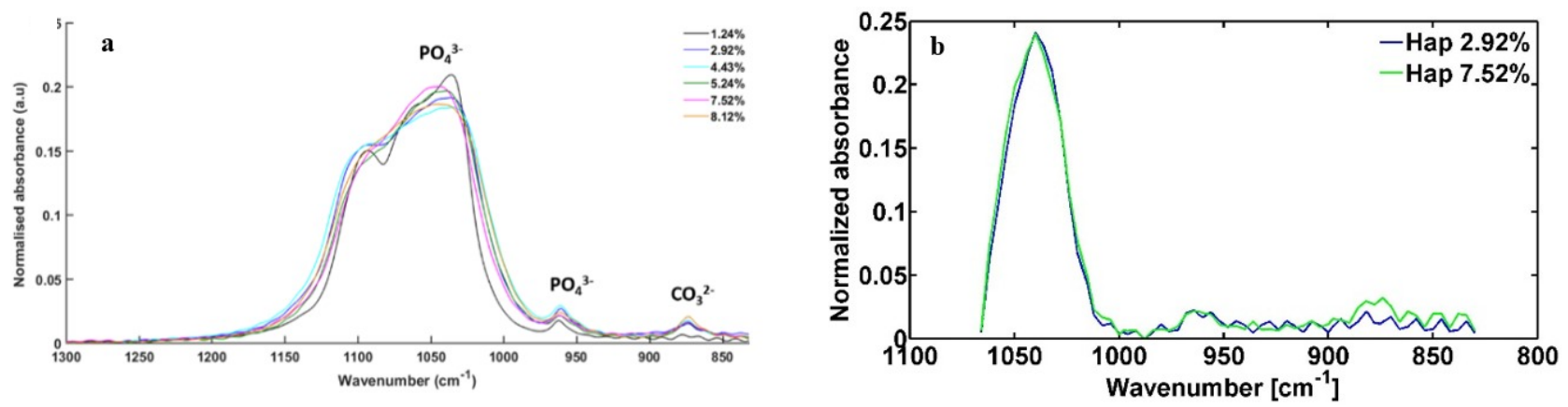

Figure 2: (a) Spectra of $\mathrm{KBr}$ pellets containing HAP powders measured using micro-FTIR Agilent system in the spectral range $1300-830 \mathrm{~cm}^{-1}$ (b) Normalized spectra of HAP with $7.52 \%$ and $2.92 \%$ carbonate substitution measured using upconversion system. 
It is noted that the absorption spectra measured by upconversion in Figure 2(b), appears noisier than that of the FTIR spectra, see Figure 2(a). In order to investigate the origin of the noise in the upconversion spectra, the absorption profile of the HAP $7.52 \%$ pellet was investigated at 881 and $920 \mathrm{~cm}^{-1}$ using the upconversion system with raster scanning.

Figure 3 shows the transmitted intensity in the upconverted images of the HAP sample with $7.52 \%$ carbonate substitution in $2 \mathrm{D}$ at the two different LWIR wavelengths. The same area was scanned at the two wavelengths, showing an interference-like pattern, however, shifted spatially. A similar pattern was observed in another pellet (not shown). The interference fringe are moving with wavelength, hence, the noise presented in the upconverted spectra is expectedly due to interference between the front and back surface of the pellet, when using a coherent illumination source like the QCL.
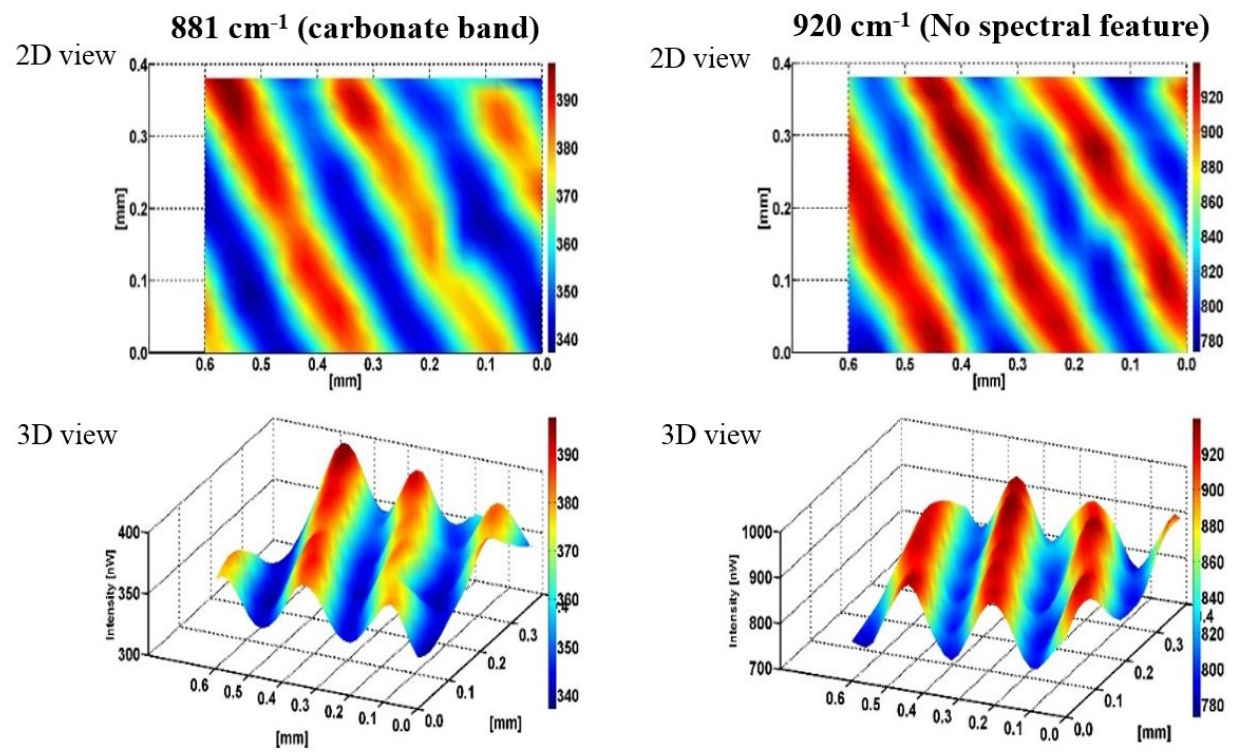

Figure 3: 2D/3D Intensity maps of HAP with $7.52 \%$ carbonate substitution measured at 881 and $920 \mathrm{~cm}^{-1}$ respectively measured using upconversion system. Note the different scales at the two wavelengths.

The HAP spectra shown in Figure 2(b) were measured at a single point in $\mathrm{KBr}$ pellets. The noise observed in upconversion spectra appears to be mainly due to interference effects rather than heterogeneity of the HAP powder in the pellet. The transmitted intensity distributions seen in Figure 3 are consistent with the use of a coherent light source to measure on a slightly wedged pellet.

\subsection{Breast cancer tissue}

Figure 4 shows the FTIR and the upconverted images of microcalcifications found in in situ breast cancer tissue. Figure 4(a) shows histological section of in situ breast cancer tissue with H\&E staining. Figure 4(b) is obtained at $1032 \mathrm{~cm}^{-1}$ using the micro-FTIR imaging system with MCT-FPA detector including 128x128 pixel arrays. Figure 4(c) shows the same tissue sample imaged by upconversion specifically selecting the wavenumber of $1020 \mathrm{~cm}^{-1}$ corresponding to the phosphate band. The upconverted image is measured by raster scanning with step size of $20 \mu \mathrm{m}$. The LWIR signal through the sample was upconverted (from $1020 \mathrm{~cm}^{-1}$ ) into NIR $(959 \mathrm{~nm}$ ) which was detected using a silicon based power meter. The upconverted image is seen to show good agreement with the FTIR image at similar wavenumber. 


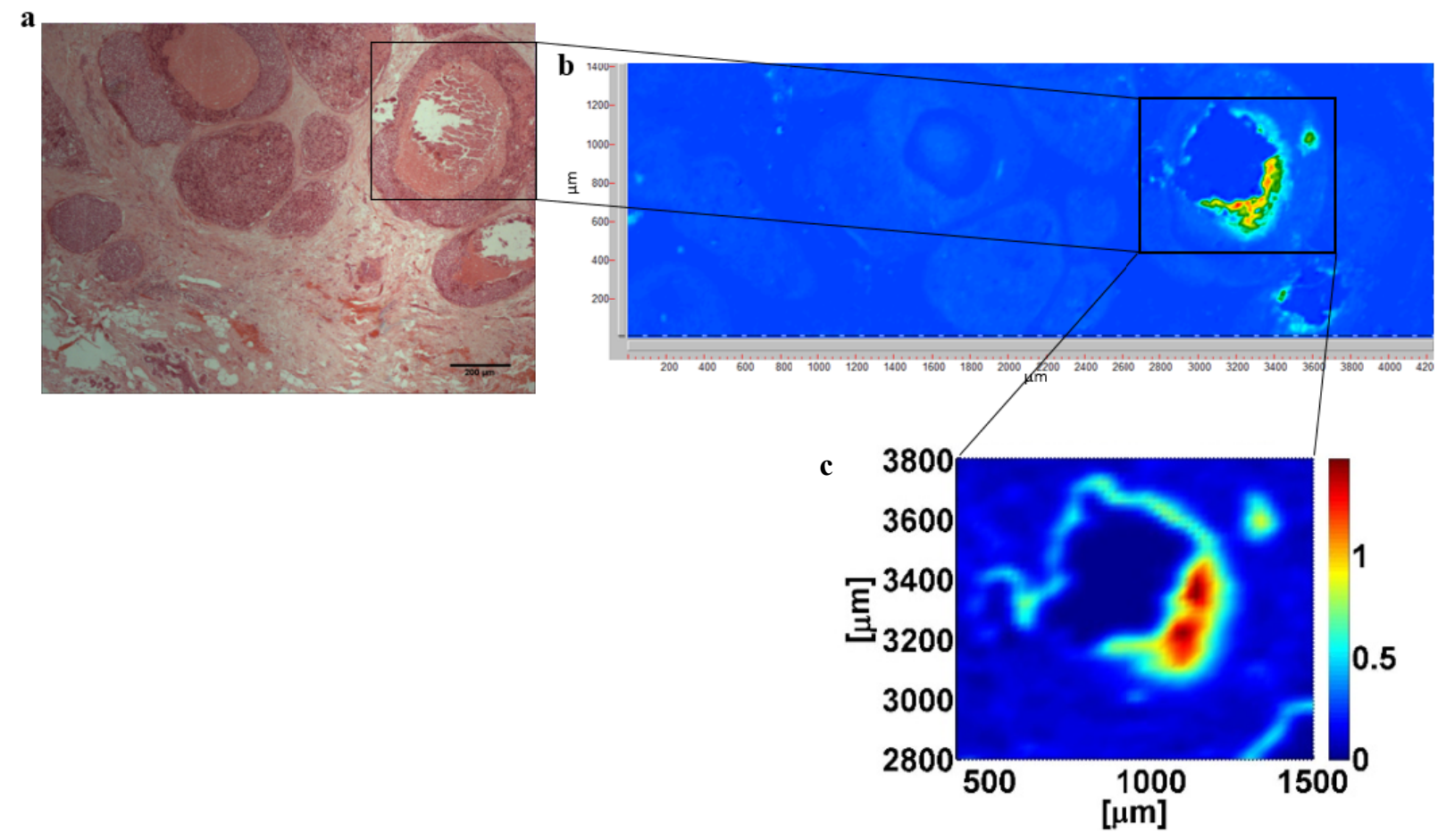

Figure 4: (a) Histological section with H\&E staining using transmission microscope with magnification $\mathrm{x} 4$ (b) FTIR image refers to the phosphate peak distribution at $1032 \mathrm{~cm}^{-1}$ with magnification x15 (pixel size: $5.5 \mu \mathrm{m}^{2}$ ) and (c) upconverted image refers to the phosphate peak distribution at $1020 \mathrm{~cm}^{-1}$ with scanned step size of $20 \mu \mathrm{m}$.

Only few discrete wavelengths are required to identify the microcalcifications for phosphate and carbonate bands at 1020 and $875 \mathrm{~cm}^{-1}$. Figure 5 shows the upconverted images obtained at the wavenumbers of $1020,975,964,920,881,875$, and $842 \mathrm{~cm}^{-1}$, respectively. These images are acquired with a raster scanning step size of $20 \mu \mathrm{m}$. It can be seen, that there is a significant decrease in signal (increased absorbance) at the phosphate absorption band in the microcalcification area (black square) compared to the surrounding. Acquiring images at other different wavelengths allows the estimation of the relative compositional contributions to the spectral absorption, thus enabling analyzed further analysis of the spectral features of microcalcifications.
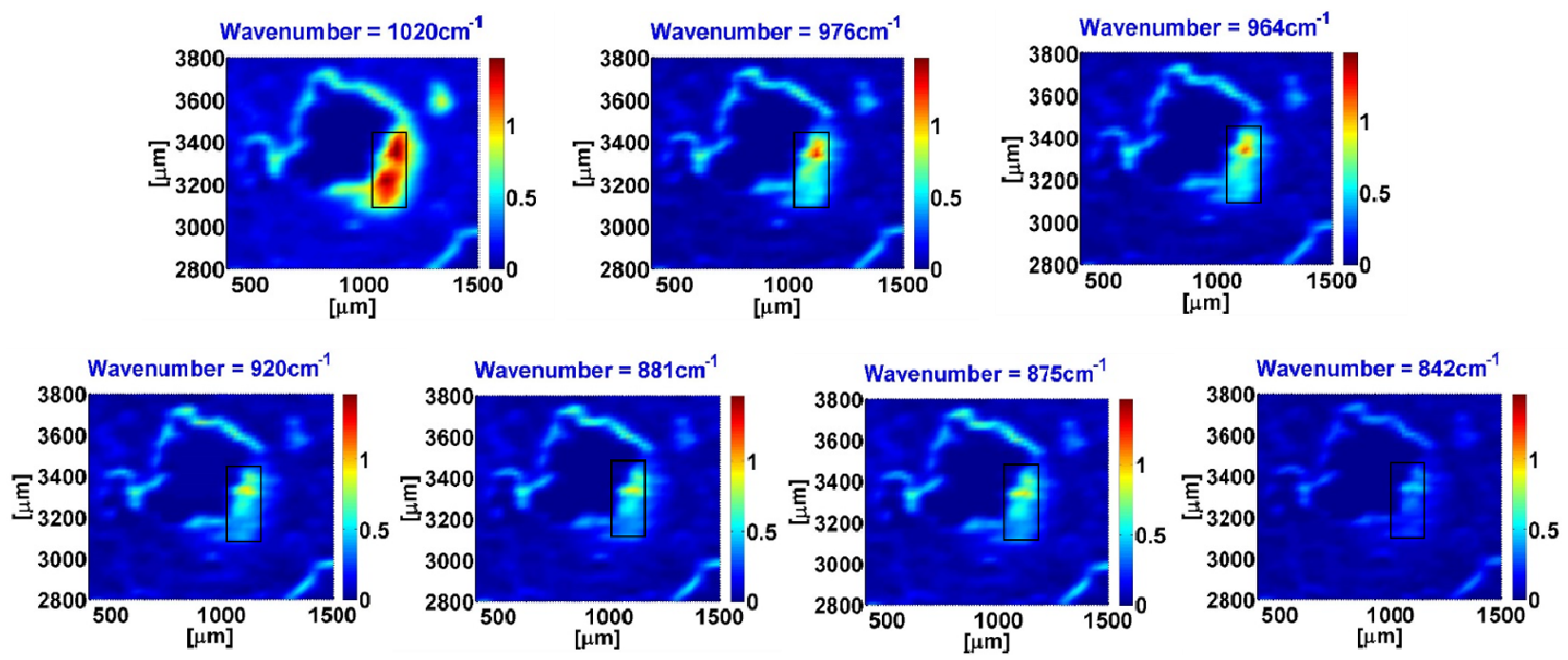

Figure 5: Upconverted images of in situ breast cancer tissue obtained at 1020,975, 964, 920,881, 875, and $842 \mathrm{~cm}^{-1}$ corresponding to the upconverted signals in the range of 959 to $976 \mathrm{~nm}$. 
The absorption spectrum is analyzed in the area where phosphate band is highly absorbing in the Figure 6(a). Figure 6(b) shows the spectrum extracted from microcalcification area (dashed square), whereas Figure 6(c) shows a spectrum extracted from surrounding tissue. The MCT-FPA detector becomes noisy in the LWIR regime $\left(900-800 \mathrm{~cm}^{-1}\right)$, therefore the signatures below $900 \mathrm{~cm}^{-1}$ cannot be considered reliable. The upconverted spectra was retrieved from a series of images (shown in Figure 5) measured at 1020, 975, 964, 920, 881, 875, and $842 \mathrm{~cm}^{-1}$, respectively. The FTIR and upconverted spectra measured from surrounding tissue demonstrate that there is only a small tissue phosphate absorption feature in this area. A single pixel (green circle) and an averaging of 6 pixels (red circle) using upconversion are compared in Figure 6 (b) and (c). A spectrum has been also extracted from FTIR image (Figure 4 b) recorded using micro-FTIR Agilent imaging system.

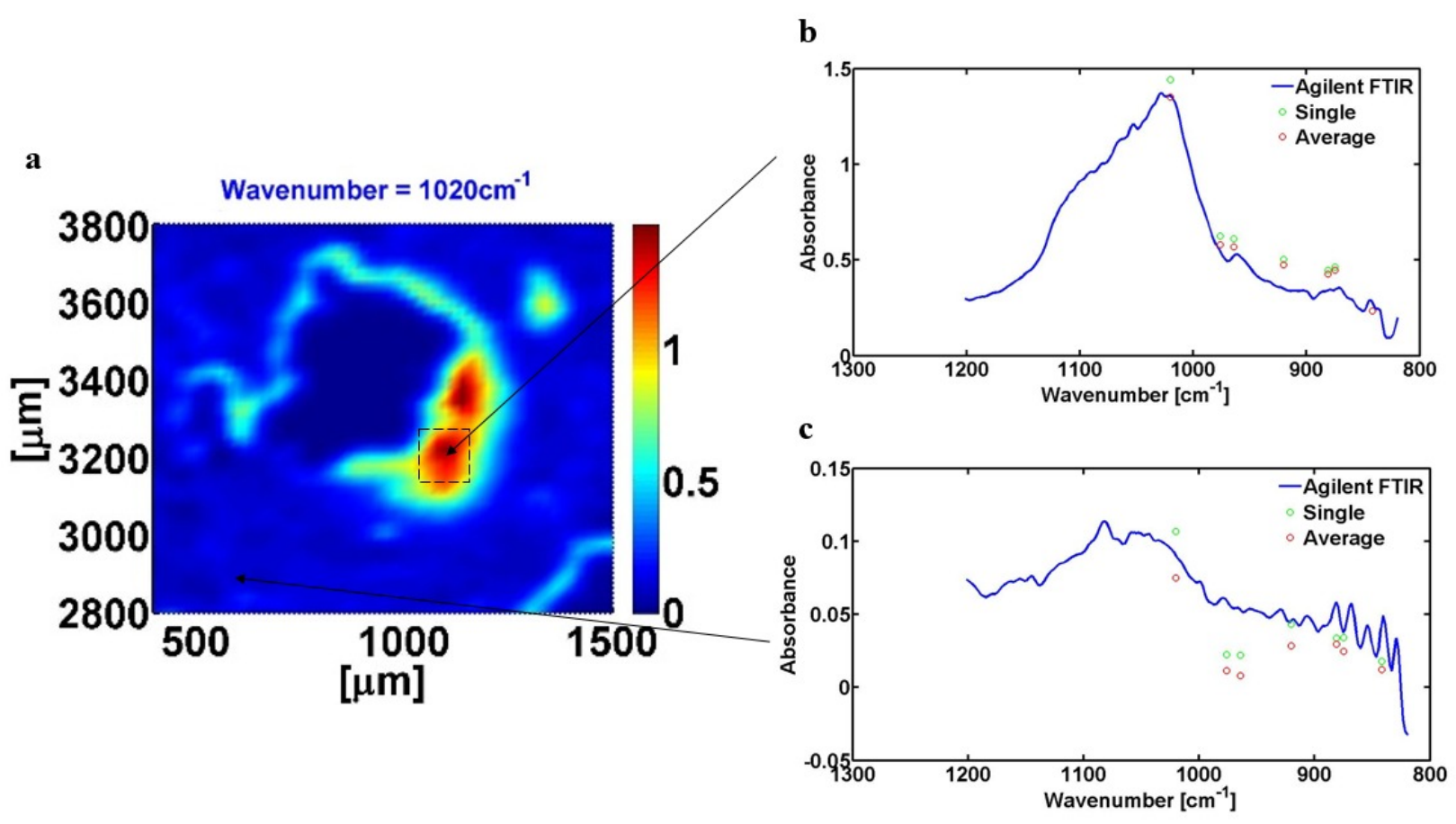

Figure 6: (a) Upconverted image at phosphate peak at $1020 \mathrm{~cm}^{-1}$ and spectra measured using micro-FTIR imaging and upconversion systems from (b) microcalcification and (c) tissue surrounding in in situ breast sample.

\section{CONCLUSION}

It is demonstrated in the study that breast cancer tissues can be analyzed using an upconversion detector with a raster scanning microscopy system at discrete wavenumbers, potentially improving the signal-to noise ratio using silicon based detectors when compared to direct detection in the LWIR range. It was possible to detect the carbonate bands at $875 \mathrm{~cm}^{-1}$ that can be challenging for the Agilent micro-FTIR imaging system to measure due to a combination of the limited number of IR photons longer than $10 \mu \mathrm{m}$ imaged on the MCT-FPA detector and its inherent sensitivity in this wavelength range. Only a few discrete wavelengths are required to identify the microcalcification from phosphate and carbonate bands at 1020 and $875 \mathrm{~cm}^{-1}$, respectively. Therefore, the combination of a tunable QCL, a raster scanning microscope, and the upconversion system shows potential for chemical imaging of tissue at selected wavelengths. The system can potentially be developed into a breast biopsy diagnostic tool for breast cancer in a clinical setting.

\section{ACKNOWLEDGMENTS}

The Mid-TECH project has received founding from the European Union Horizon 2020 research innovation programme under Grant Agreement No. 642661.

We thank Prof Mary Tecklenburg for providing us carbonated hydroxyapatite powers. 


\section{REFERENCES}

[1] DeSantis C. E., Ma J., Sauer A.G., Newman A.L., Jemal A. "Breast Cancer Statistics, 2017, Racial Disparity in Mortality by State," CA Cancer J Clin 67, 439-448 (2017).

[2] Baker R., Rogers K. D., Parker A.W., Stone N. "New relationships between breast microcalcifications and cancer," British Journal of Cancer. 103, 1034-1039 (2010).

[3] Pleshko N., Boskey A., Mendelsohn R. "Novel infrared spectroscopic method for the determination of crystallinity of hydroxyapatite minerals," Biophysical journal. 60, 786-793 (1991).

[4] Kerssens M.M., Matousek P., Rogers K., Stone N. "Towards a safe non-invasive method for evaluating the carbonate substitution levels of hydroxyapatite (HAP) in microcalcifications found in breast tissue," Analyst. 135, 3156-3161 (2010).

[5] Rogalski A., Infrared Detectors, CRC Press, 2010.

[6] Dam J. S., Pedersen C., Tidemand-Lichtenberg P., "Room temperature mid-IR single photon spectral imaging" Nature Photonics, 6, 788-793 (2012).

[7] Tidemand-Lichtenberg P., Dam J. S., Andersen H. V., Høgstedt L., and Pedersen C., "Mid-infrared upconversion spectroscopy” J. Opt. Soc. Am. B 33(11), D28 -D35 (2016). 